\title{
Young Magellanic Cloud Clusters ( $<1$ Gyr): Census, Properties, Star Formation History
}

\author{
Eva K. Grebel, Dennis Zaritsky, Jason Harris \\ UCO/Lick Observatory, UCSC, Santa Cruz, CA 95064, USA
}

\author{
Ian Thompson \\ Observatories of the Carnegie Institution, 813 Santa Barbara St., \\ Pasadena, CA 91101, USA
}

\begin{abstract}
We report preliminary results from an automated cluster survey of the Magellanic Clouds aimed at improving the cluster census and at deriving cluster properties from their resolved stellar content. The survey is tripling the number of known clusters. The cluster age distribution shows similar peaks at $100-200 \mathrm{Myr}$ in LMC and SMC, coincident with the closest encounter of the Clouds and perigalacticon.
\end{abstract}

\section{Introduction}

In contrast to the Milky Way, the Magellanic Clouds contain a plethora of compact star clusters of all ages. Photographic surveys led to the detection of $\sim 2400$ clusters in the LMC and $\sim 700$ clusters in the SMC, while the true number of star clusters is estimated to be more than twice as high (Westerlund 1997).

The new large-scale CCD surveys of the Magellanic Clouds provide homogeneous multi-color coverage of the Clouds with good spatial resolution and are well-suited to improve the census of star clusters. We are using data from the Magellanic Clouds Photometric Survey (Zaritsky et al. 1997) and from the Optical Gravitational Microlensing Experiment (Udalski et al. 1998) for this purpose. These surveys also allow us to derive ages and structural parameters based on the resolved stellar content of the clusters.

\section{Search Methodology}

Our search for clusters uses a minimum spanning tree algorithm to identify areas of higher stellar density (Zahn 1971). The algorithm finds clusters by searching for minima in the average distance between any two stars. The user specifies the allowable standard deviation of the average distance and a minimum number of stars per "cluster". Without these constraints the algorithm will find extended strings of stars as clusters, or consider any two close stars a "cluster".

In addition to the density criterion we make use of the magnitude and color information in our dataset. The data are divided into subsets covering certain magnitude ranges and color ranges representing different evolutionary 
phases. We require detection in at least two of these subsets to consider a density enhancement to be a star cluster candidate. For instance, a density enhancement detected in the red clump subset and the subset of faint mainsequence stars is a good candidate for an intermediate-age cluster. Similarly, a density enhancement detected in the blue and in the bright subsamples stands a good chance of indicating a young cluster. We use five such color-magnitude selections of subregions representing certain evolutionary phases plus the full data set.

After fine-tuning the cluster algorithm criteria for each subset, $94 \%$ of the known star clusters could be retrieved. Clusters that escaped detection were either objects within OB associations with displaced or poorly defined centers, or objects that were not visible in our data even when visually inspecting our frames (possibly plate errors in the earlier photographic surveys?).

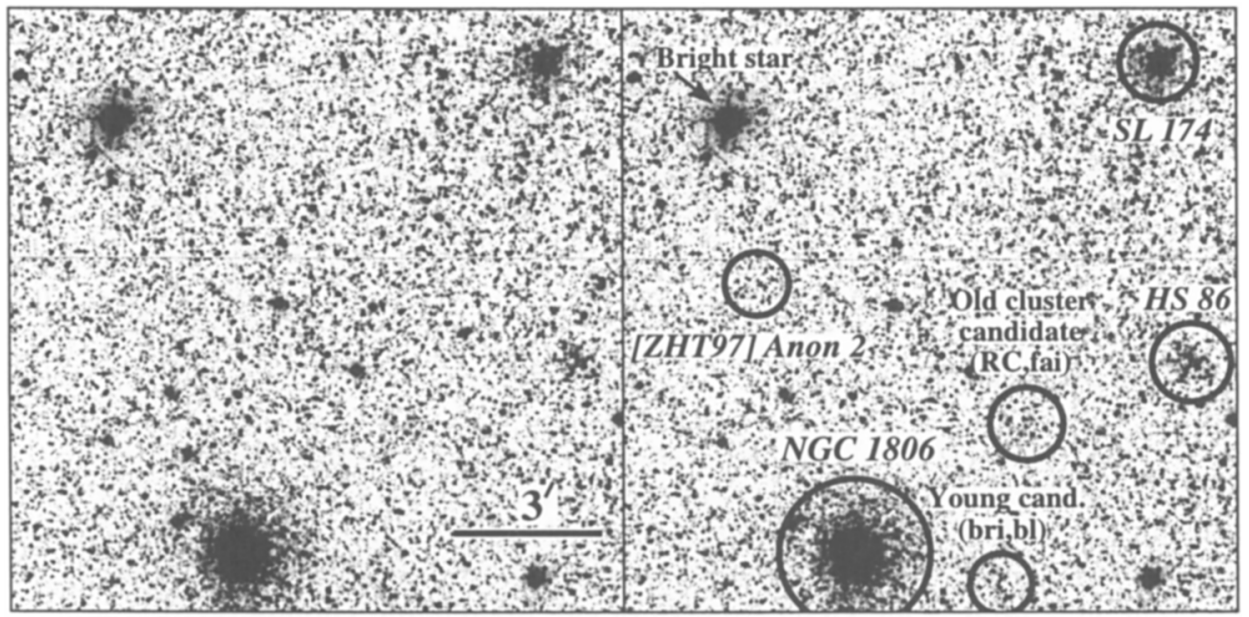

Figure 1. Cluster search results. Left: Small field from the Magellanic Clouds Photometric Survey (Zaritsky et al. 1997). Right: Same field with marked cluster detections. For new candidates the magnitude/color-selected subsets in which they were detected are specified (bri=bright, $b l=b l u e$, fai=faint, $\mathrm{RC}=$ red clump).

\section{Monte-Carlo Simulations}

Monte-Carlo simulations were run to assess the reliability of our detections on artificial fields with randomly distributed stars $\left(10^{6}\right)$ and fields with artificial density gradients. The density in both Monte-Carlo sets was chosen to reproduce the observed densities in our data away from and near to the LMC bar. Furthermore, we created fields with artificial clusters $(14,000)$. Observed magnitudes were randomly assigned to the Monte-Carlo star positions, and magnitude/colorselected subsets were extracted as before. 


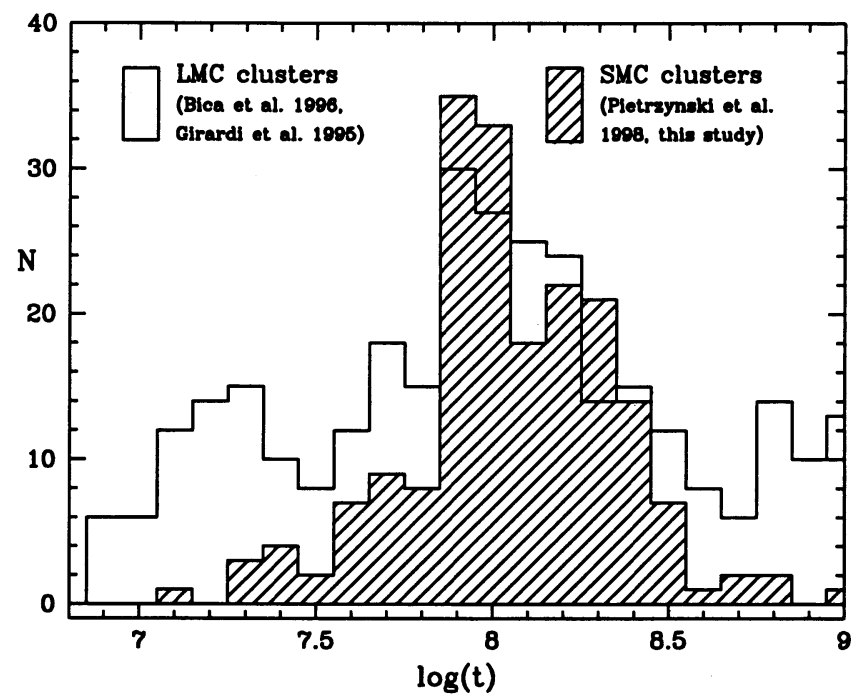

Figure 2. The age distributions of LMC and SMC clusters both peak around $\log (\mathrm{t})=8$, when the Clouds had their closest encounter and last perigalacticon.

On average we found $10-20 \%$ spurious detections in each of the magnitude/ color-selected subsets with a slight increase of $2-3 \%$ in fields with artificial density gradients. Spurious cluster candidate detections were reduced to $5 \%$ when we imposed the requirement of independent detection in at least two subsets. About $60 \%$ of the artificial clusters with $\geq 30$ members and $\sim 100 \%$ of clusters with $\geq 50$ stars were detected by the minimum spanning tree algorithm, which demonstrates its high sensitivity.

\section{Search Results}

In the areas of the Magellanic Clouds Photometric Survey searched so far the number of cluster candidates was tripled. Among the new clusters are a few bright amorphous agglomerates in extended OB associations with ages of mostly $<30 \mathrm{Myr}$. Many sparse, bright concentrations of OB stars were detected as cluster candidates (ages < $40 \mathrm{Myr}$ ). These objects resemble the multiple, unbound compact cores of $\mathrm{OB}$ associations that will disperse quickly (van den Bergh, this volume). Finally, the new detections comprise many faint, sparse clusters with estimated ages $>600 \mathrm{Myr}$. Our new detections seem to represent a continuum of open clusters in various stages of dissolution ranging from sparse young objects that will be unobservable after $>100 \mathrm{Myr}$ to initially more compact agglomerates that require $\gg 100 \mathrm{Myr}$ to disperse.

A preliminary analysis of structural parameters indicates that few loose clusters with radii $>40^{\prime \prime}$ are detectable after $200 \mathrm{Myr}$, while slightly more compact clusters with radii $<30^{\prime \prime}$ are easily detected until $\sim 400 \mathrm{Myr}$. Older clusters tend to have higher concentration parameters. 


\section{Age Distribution and Interactions}

Isochrone-based ages using both Geneva and Padua models were derived for a complete sample of $\sim 200$ populous star clusters from the catalog of Pietrzyński et al. (1998) in the central $\sim 2.4$ degrees $^{2}$ of the SMC. The resulting age distribution shows a pronounced peak at $\sim 100 \mathrm{Myr}$ and a second, smaller peak at $\sim 200 \mathrm{Myr}$ (Figure 2). These two peaks resemble the broad peak around 100-200 Myr in age distribution of LMC clusters found by Girardi et al. (1995) from an age calibration of integrated colors.

Geisler et al. (1997) showed that ages for intermediate-age and old clusters derived from integrated colors can be significantly $(8-10 \mathrm{Gyr})$ in error due to stochastic effects by bright field stars and crowding. Comparing our isochrone ages for clusters younger than 1 Gyr to integrated-color ages for the same SMC clusters from Elson \& Fall (1985) we find good overall agreement despite considerable scatter $(\log (\Delta t) \pm 0.3)$, indicating that for bright young clusters the errors are less significant.

The SMC data do not show a period of enhanced cluster formation at more recent times $(\log (\mathrm{t}) \sim 7)$, nor a pronounced peak at $\log (\mathrm{t}) \sim 9.1$, as seen in the LMC.

N-body simulations (Gardiner et al. 1994) suggest that the last close LMC/ SMC encounter was $\sim 200 \mathrm{Myr}$ ago, and that the last perigalacticon occurred $\sim 100 \mathrm{Myr}$ ago. The common peaks in the cluster age distribution at $\log (\mathrm{t}) \sim 8$ may be due to an enhanced cluster formation rate triggered by these events, while interaction between the Clouds does not seem to have been the cause of the increased cluster formation in the LMC seen at earlier and later times.

Acknowledgments. Support for this work was provided by NASA LTSA grant NAG-5-3501, NSF AST-96 19576, the Packard Foundation, and the Sloan Foundation.

\section{References}

Bica, E.L.D., Clariá, J.J., Dottori, H., Santos, J.F.C., \& Piatti, A.E. 1996, ApJS, 102,57

Elson, R.A.W., \& Fall, S.M. 1985, ApJ, 299, 211

Gardiner, L.T., Sawa, T., \& Fujimoto, M. 1994, MNRAS, 266, 567

Geisler. D., Bica, E., Dottori, H., Clariá, J.J., Piatti, A.E., \& Santos, J.F.C. 1997, AJ, 114, 1920

Girardi, L., Chiosi, C., Bertelli, G., \& Bressan, A. 1995, A\&A, 298, 87

Pietrzyński, G., Udalski, A., Kubiak, M., Szymański, M., Woźniak, P., \& Żebruń, K. 1998, AcA, 48,175

Udalski, A., Szymański, M., Kubiak, M., Pietrzyński, G., Woźniak, P., \& Żebruń, K. 1998, AcA, 48, 147

Westerlund, B.E. 1997, The Magellanic Clouds, Cambridge University Press

Zahn, C.T. 1971, IEEE Trans. on Computers, C-20, 68

Zaritsky, D., Harris, J., \& Thompson, I. 1997, AJ, 114, 1002 


\section{Discussion}

John Gallagher: With regard to the long lives of star-forming regions, it might be interesting to note that Robbie Dohm-Palmer found a similar behaviour in HST observations of the dI galaxy Sextans A (Dohm-Palmer et al. 1998, AJ). He used blue loop stars for his age tracers and showed that star-forming regions have lifetimes of $>100 \mathrm{Myr}$.

Grebel: Indeed this is very similar to what we find in the LMC from supergiants and Cepheids. We see long-lived chains of star formation that were active for $\approx$ $200 \mathrm{Myr}$. Interestingly these areas appear to correspond to the earlier proposed asymmetric "spiral arms" in the LMC. Gardiner and collaborators carried out $\mathrm{N}$-body simulations to study the effect of an off centered bar on a rotating disk and showed that this leads to the formation of a non-density wave "spiral arm" and sites of star formation along this feature. The resulting picture looks very similar to the observed global star formation patterns across the face of the LMC disk. It would seem possible that the bar of Sextans A, which appears to be off centered, may have a similar effect in Sex A by confining gas along what develops into long-lived chains of star formation.

David Graff: What was the source for your Cepheids?

Grebel: The catalogue of Payne-Gaposhkin and the General Catalogue of Variable Stars (Kholopov 1995, 1997).

David Graff: How does the Drift-scan survey compare with the OGLE survey?

Grebel: The Magellanic Clouds Photometric Survey will cover $8^{\circ} \times 8^{\circ}$ of the LCM and the central $4^{\circ} \times 4^{\circ}$ of the SMC in $U B V I$. The goal of this drift-scan survey is to provide multicolor photometry down to $V=21 \mathrm{mag}$ for stellar population and star formation history studies.

The OGLE survey concentrates on the bar regions of the Magellanic Clouds and covers them in $B V I$ searching for microlensing events and other signs of variability. 